\title{
LA GÉOGRAPHIE, ÇA PEUT SURVIVRE À LA GUERRE CORRESPONDANCE ENTRE JEAN GOTTMANN ET ORLANDO RIBEIRO*
}

\author{
SuZanne Daveau ${ }^{1}$
}

\begin{abstract}
Résumé - On montre l'intérêt de la correspondance privée, échangée entre 1938 et 1973 par deux grands géographes. Elle fait revivre des épisodes tragiques de l'Histoire du XXe siècle, ainsi que la lutte passionnée de certains chercheurs pour maintenir cependant leur activité. L'information est particulièrement abondante pour l'époque 1939/43, quand commença et se généralisa la II Guerre Mondiale, grâce à l'abondant courrier alors envoyé par Jean Gottmann (1915/1994) à Orlando Ribeiro. On y voit renaître successivement l'activité du prestigieux Institut de Géographie de Paris, l'exode vers le Sud de la France, face à l'invasion allemande, la fuite des juifs à travers le Portugal, l'organisation transatlantique d'une Géographie mise au service de la lutte contre les nazis puis de la reconstruction de l'Europe. L'intéressante personnalité de Jean Gottmann apparaît bien dans ses lettres. Les diverses publications qui viennent de lui être consacrées, aideront à replacer l'étude de son œuvre entre les sources d'inspiration les plus utiles aux jeunes géographes.
\end{abstract}

Mots-clés: Jean Gottmann, Orlando Ribeiro, histoire de la Géographie, II Guerre Mondiale, correspondance.

Resumo - A GEOGRAFIA PODE SOBREVIVER À GUERRA. CORRESPONDÊNCIA ENTRE JeAn GotTmann e Orlando Ribeiro - Mostra-se o interesse da correspondência privada, trocada de 1938 a 1973 entre dois mestres da Geografia, que permite voltar a dar vida a episódios trágicos da História do século XX e à luta apaixonada de alguns cientistas para manter, no entanto, a sua actividade. A informação é particularmente abundante em relação ao período 1939/43, quando deflagrou e alastrou a II Guerra Mundial, graças ao abundante correio então mandado por Jean Gottmann (1915/1994) a Orlando Ribeiro. Renascem sucessivamente a actividade do prestigiado Institut de Géographie de Paris, o êxodo para o Sul da França frente à invasão alemã, a fuga dos judeus através de Portugal, a organização transatlântica de uma Geografia posta ao serviço da luta contra o nazismo e, depois, a reconstrução da Europa. A aliciante personalidade de Jean Gottmann transparece através das suas

* Recebido: 4/06/2007. Revisto: 20/06/2007. Aceite: 21/07/2007.

1 Investigadora do Centro de Estudos Geográficos da Universidade de Lisboa. E-mail: sdaveau@clix.pt 
cartas. Várias publicações recentes, que lhe foram dedicadas, devem incitar a introduzir de novo o estudo da sua obra entre as fontes de inspiração mais úteis aos jovens geógrafos.

Palavras-chave: Jean Gottmann, Orlando Ribeiro, história da Geografia, II Guerra Mundial, correspondência.

\begin{abstract}
GEOGRAPHY CAN SURVIVE THE WAR. CORRESPONDENCE BETWEEN JEAN GotTMANn AND ORLANDO RIBEIRO - This article highlights the interest of the private correspondence exchanged between 1938 and 1973 by two major geographers. It brings back to life several tragic episodes of the history of the 20th Century, as well as the passionate struggle by some scientists to remain active in their field throughout. In particular, there is a wealth of information with regard to the period 1939-43 , when World War II broke out and spread, thanks to the numerous letters sent by Jean Gottmann (1915-1994) to Orlando Ribeiro. One after the other, references can be found there to the activity of the renowned Institut de Géographie in Paris, the exodus to Southern France at the time of the German invasion, the flight of the Jews through Portugal and the transatlantic organisation of a Geography aiming to serve the fight against Nazism and, later, the reconstruction of Europe. Jean Gottmann's fascinating personality emerges clearly from his letters. Several recent publications that have focused on him will certainly contribute to reintroducing the study of his life and work as a most useful source of inspiration for young geographers.
\end{abstract}

Key words: Jean Gottmann, Orlando Ribeiro, history of Geography, World War II, correspondence.

Les Actes du Colloque International L'Orbite de la Géographie de Jean Gottmann, qui s'est tenu à Paris en mars 2005, viennent d'être publiés sous la forme d'un numéro spécial de La Géographie. Ce volume de 310 pages réunit 25 contributions et un important texte inédit de Jean Gottmann. Il réunit une documentation abondante et de qualité sur le parcours et l'œuvre d'un des très grands géographes du XXe siècle. Le colloque a été réalisé conjointement par la Sorbonne, la Bibliothèque Nationale de France et la Société de Géographie, mais sa préparation et sa publication sont dues principalement aux Professeurs Calogero et Luca Muscarà. En 2003, le premier avait édité 39 contributions, regroupées sous le titre In the steps of Jean Gottmann. Quant à l'ouvrage de Luca Muscarà, La Strada di Jean Gottmann, il date de 2005.

En 1999, alors que je commençais à classer les manuscrits et les lettres de mon mari, Orlando Ribeiro, pour les mettre en condition d'être légués, comme il l'avait désiré, au Centro de Estudos Geográficos (Centre d'Études Géographiques de Lisbonne) (Daveau et al., 2002), j'avais découvert des lots de lettres anciennes qui projetaient une lueur émouvante sur les conditions dans lesquelles les membres de l'Institut de Géographie de Paris avaient vécu les heures tragiques de l'invasion de la France, en 1940. En effet, Orlando Ribeiro, rentré au Portugal au moment même où Paris allait être occupé par les troupes allemandes, servait alors de 'boîte postale' pour les collègues et amis dispersés, qui tentaient déses- 
pérément de rétablir des contacts familiaux et de se raccrocher à l'activité scientifique qui avait été leur raison d'être. Entre ces lettres, celles de Jean Gottmann sont particulièrement nombreuses et intéressantes. J'avais alors correspondu avec le Professeur Luca Muscarà, qui venait de participer, à Lisbonne, au Symposium sur Voyage, Circulation et Transfert d'Idées Géographiques, dans le cadre de la Conférence Régionale 98 de l'U.G.I. (Finisterra, 1998), et nous avions échangé les photocopies de quelques lettres de Jean Gottmann et de mon mari.

Un lapsus d'organisation du Colloque parisien, plus d'une fois remis et toujours attendu, fit que j'appris trop tard sa réalisation. La très intéressante documentation aujourd'hui publiée sur la vie et l'œuvre de Jean Gottmann (1915/1994) m'incite à proposer, en une sorte d'annexe aux Actes de ce Colloque, la présente note sur les relations qu'entretinrent deux grands géographes, contemporains et amis. Elle est basée principalement sur leur correspondance et me paraît démontrer, une fois de plus, combien il est important de préserver et de rendre disponibles ces témoignages de l'activité des grands esprits qui nous ont précédés. Rien de plus vivant, de plus spontané et de plus riche que ces notations personnelles et instantanées, où surgissent les projets, les doutes, les espoirs ou désespoirs, des auteurs dont nous découvrons chaque jour la persistante actualité.

La correspondance de Jean Gottmann réunit environ 15000 lettres, échangées de 1933 à 1994 avec pas moins de 2651 correspondants. Elle est désormais confiée au Département des Cartes et Plans de la Bibliothèque Nationale de France (Sarazin, 2007), dans le cadre du 'fonds Gottmann' offert par sa femme, Bernice Adelson. La correspondance d'Orlando Ribeiro (1911/1997) appartient, par don testamentaire de celui-ci, au Centro de Estudos Geográficos de Lisbonne (Daveau et al., 2002). Les lettres qui étaient conservées à notre domicile ont déjà subi un classement provisoire et vont être confiées à l'Arquivo da Cultura Portuguesa Contemporânea de la Biblioteca Nacional de Portugal. Il est hautement souhaitable que l'abondante correspondance dite officielle, conservée par le Centro de Estudos Geográficos, soit, sans trop de délais, rendue elle aussi accessible dans le cadre des archives scientifiques de ce Centre.

La correspondance Gottmann-Ribeiro, retrouvée à ce jour, va de 1938 à 1973. On peut la diviser en cinq périodes d'importance et de durée inégales:

- Le séjour commun à Paris, où deux jeunes géographes se formaient avec enthousiasme, sous la direction de E. de Martonne et A. Demangeon;

- La tourmente des années 1940/41, où les lettres se multiplient jusqu'à l'embarquement de Jean Gottmann pour New York, le 11 novembre 1941 à Lisbonne;

- Les quelques lettres échangées à travers l'Atlantique en 1942/43;

- La reprise de la correspondance à l'époque du Congrès Géographique International de Lisbonne, en 1949;

- Les quelques lettres postérieures. 
L'essentiel de cette correspondance se situe donc au cours de la partie la moins documentée de la vie scientifique de Jean Gottmann, celle qui antécède sa fuite en Amérique, en 1941. En effet, pour parvenir alors à quitter l'Europe, «il fallait voyager léger» (Sarazin, 2007: 25).

\section{LES PREMIERS CONTACTS PARISIENS}

Jean Gottmann, orphelin et réfugié d'origine russe (de surcroît, juif et apatride), était installé à Paris avec ses tuteurs depuis 1921 et il fréquenta l'Institut de Géographie de la rue Saint-Jacques à partir de 1932. Ayant présenté en 1934 un diplôme d'études supérieures sur l'Irrigation en Palestine, il devint l'assistant scientifique d'Albert Demangeon. Quant à Orlando Ribeiro, un jeune Portugais déjà Docteur en Géographie par l'Université de Lisbonne, il débarquait à Paris, en janvier 1937, comme Lecteur de Portugais à la Sorbonne, mais surtout pour y compléter sa formation de géographe.

L'Institut de Géographie de Paris était alors le haut lieu de la Géographie, non seulement française mais internationale. Emmanuel de Martonne, gendre de Paul Vidal de La Blache, le fondateur de la Géographie moderne en France, était un grand organisateur, à la fois craint et respecté. Il partageait la direction de l'Institut avec Albert Demangeon, chargé de gérer la Géographie humaine, tandis que de Martonne, auteur du célèbre Traité de Géographie Physique, régentait la Géographie physique. Une insidieuse fêlure s'était ainsi déjà installée, menaçant l'unité de notre discipline, fêlure encore peu apparente et peu nocive, puisque tous les géographes avaient encore reçu une formation générale, mais qui allait se révéler fort néfaste au cours des décennies suivantes.

Les deux jeunes gens sympathisèrent et, le 16 août 1938, alors que l'un et l'autre avaient participé, en juillet, à l'excellent Congrès International de Géographie d'Amsterdam, Gottmann envoyait d'Aix-les-Bains, où il passait ses vacances, une carte postale à Orlando pour le «remercier vivement pour votre compagnie qui m'a rendu bien plus agréable et intéressant le séjour d'Amsterdam», tout en évoquant les vacances «laborieuses» que son ami passait alors au Portugal, ainsi que son retour projeté à Paris. Mais, dès le 2 octobre, une autre lettre, répondant à celle qu'Orlando lui avait envoyée de la Beira Baixa où il préparait sa thèse d'État, présageait la tourmente à venir: «L'alerte passée (...), la détente nerveuse a été si vive que les manifestations populaires ont pris l'allure d'un triomphe. Pour ma part, j'en suis resté très triste et ne puis me défaire d'un pessimisme très noir. Il me semble (...) qu'il y a eu défaite et même peu honorable; en somme, un sursis». La France et l'Angleterre venaient en effet de capituler diplomatiquement à Munich, le 29 septembre, devant les exigences d'Hitler, en acceptant le démembrement de la Tchécoslovaquie. L'alerte avait été si vive que tous les jeunes français mobilisables avaient été rappelés, de sorte que l'assistant Pierre Birot, qui se trouvait alors au Portugal, pour y com- 
mencer des recherches géomorphologiques, avait dû rentrer précipitamment en France. Le 9 octobre, il écrivait à Orlando Ribeiro: «J'ai enfin déposé l'uniforme et aussitôt de retour à Paris je me suis mis à classer mes notes de voyage».

Un an plus tard, l'atmosphère était devenue encore plus angoissante. Le 29 août 1939, Gottmann écrivait qu'il se passait «en Europe des choses qui remettent tout en question: le Congrès [International de Géographie, prévu pour 1942 à Lisbonne] comme votre rencontre avec Birot au Portugal. (...) Nous sommes prêts à tout sans broncher et Paris, déjà, vit dans l'atmosphère guerrière (...). J'espère que vous serez bien sur votre terrain de thèse en septembre et que moi j'irai au Maroc [avec une bourse pour préparer une thèse projetée sur l'irrigation dans les pays méditerranéens]. $M a$ cabine était commandée déjà sur le bateau qui part samedi de Marseille. Je vais annuler cela demain s'il n'y a rien de très nouveau, dans le sens favorable (...). Pour ma part j'attends une mobilisation générale pour recevoir une affectation militaire quelconque [il venait d'être naturalisé Français] et je me dépêche de terminer maintenant un travail commencé sur les matières premières et d'avancer la structure agraire». Trois jours plus tard, les troupes allemandes entraient en Pologne et la guerre éclatait entre le Reich et les alliés franco-anglais.

Le 29 septembre, Gottmann écrivait à Orlando pour lui dire qu'il avait «été profondément touché par votre décision de revenir cette année à la Sorbonne (...). Pour ma part je n'ai encore aucune idée de ce que je vais devenir (...). J'attends donc et j'essaye de travailler (...). La Sorbonne doit rester à Paris et l'Institut rouvrira sans doute normalement mais avec un programme de cours réduit. Déjà Cholley et Robequain sont partis [deux des professeurs, mobilisés]». Quant à Pierre Birot, qui était à nouveau au Portugal depuis le 2 août, il allait encore rentrer avec «précipitation», comme il l'écrivait le 9 décembre à Orlando, depuis la ville de garnison où il supportait avec ennui les longs mois de cette 'drôle de guerre', pendant laquelle les armées ennemies restèrent face à face, sans encore attaquer. La situation était si calme que Birot espérait encore rencontrer Orlando, pour discuter de leurs recherches, «car je pense venir à Paris en permission vers le 5 ou 6 janvier»».

La vie continuait en effet à Paris et l'enseignement de la Géographie se poursuivait tant bien que mal, en l'absence de deux professeurs et de l'unique assistant, mobilisés. Gottmann, non appelé à cause de sa santé fragile, assurait, en compagnie du Portugais et d'un Égyptien, un minimum d'enseignement, sous la direction de Emmanuel de Martonne. Le 13 février, il avisait par un mot Orlando qu'était prévue pour le lendemain «une réunion préparatoire où nous nous entendrions sur les échantillons [de roches volcaniques à présenter aux étudiants], Mr de Martonne (...), vous, Awad et moi»».

Encore une lettre de Paris, datée du 8 juin 1940 et répondant à une missive d'Orlando, parvenue d'Hendaye. Celui-ci avait en effet quitté la France par le train, alors que l'offensive allemande déferlait depuis le 10 mai, à travers la Hollande et la Belgique, l'armée belge ayant capitulé le 28 mai. Gottmann se 
force encore à plaisanter: «vous devez faire de la géographie pacifiquement dans Lisbonne bien éclairée. Cela doit vous faire une curieuse impression. Quant à moi je continue bien tranquillement ma structure agraire et mes autres travaux du moment comme par le passé. Nous avons bien été bombardés l'autre lundi et même assez sérieusement (...) ma famille et tout le personnel de l'Institut sont intacts». Gottmann veut croire pourtant que «la victoire sera non seulement certaine mais proche». (En fait, l'armée allemande entrera dans Paris six jours plus tard, le 14 juin). Gottmann annonce être sans nouvelles de Birot: "Il y a lieu de croire qu'il soit prisonnier» et il ajoute: «Je vais voir Monsieur Demangeon qui est encore un peu souffrant mais commence à se lever». Enfin, dans un petit P.S.: «Ne faites pas votre texte pour l'A.G.F. [le Bulletin de l'Association de Géographes Français] trop long; nos revues scientifiques sont encore réduites de moitié.»

\section{LA DÉBÂCLE}

L'armée française ne parvint pas à résister à l'offensive allemande. Dès le 15 juin, les troupes allemandes traversaient la Loire, que les Français avaient encore espéré utiliser comme ultime ligne de défense. L'armistice sera signé le 22 juin. Les conditions imposées divisaient la France en deux parties, la moitié nord et toute la façade atlantique étant 'occupées', alors que le reste du pays constituait la 'zone libre'.

Les six longues lettres que Jean Gottmann envoie à Orlando de Montpellier, du 22 juin 1940 au 9 avril 1941, sont parmi les plus intéressantes qui aient été conservées. Dans la première, il évoque d'abord pudiquement ce que fut pour lui 1' 'exode', cette fuite éperdue qui entraîna vers le Sud de la France la foule des réfugiés mêlés aux soldats en déroute et que les avions allemands bombardaient un peu au hasard. Ayant quitté Paris le 11 juin dans l'aprèsmidi, il passait par Le Mans et par Vichy, en empruntant de successifs trains de marchandise, et retrouvait sa famille à Montpellier neuf jours plus tard (Muscarà, 2005: 71-73): "Pourquoi suis-je venu ici? Je n'en sais rien; j'étais porté depuis quatre jours par un flot humain irrésistible et épouvantable. Ici, dans une grande ville universitaire, nous avons décidé de rester, pour attendre, et parce que mes parents [une tante et son mari, son grand-père] $n$ 'ont vraiment pas la force de continuer. Mon itinéraire a été étrange (...). Ce que j'ai vu dépasse l'imagination d'avant juin 1940. Les yeux encore pleins de visions apocalyptiques, je crois bien avoir touché le fond de la misère morale (...). Je ne vous en dirai pas plus. Je suis heureux pour vous que vous soyez rentré chez vous à temps (...). Voici maintenant quelques nouvelles de nos maîtres. Mr de Martonne a quitté Paris le 12 aussi, emmenant dans sa voiture sa famille et il est allé dans sa propriété (Rusquerolles par Boissézon, Tarn); j'espère qu'il est bien arrivé». Mais Albert Demangeon est resté malade à Paris, avec sa femme. "Mes efforts pour lui trouver un moyen de transport 
convenable ont été vains (...). De Birot aucune nouvelle (...). Pour ma part je n'ai aucun projet d'avenir. Je ne crois pas qu'il reste de la place dans le monde de demain pour des intellectuels de mon espèce. Où aller?»

Un mois plus tard, Gottmann a moralement récupéré. Le 20 juillet, il remercie Orlando pour sa réponse et pour son «offre d'aide spontanée, faite avec tant de délicatesse et d'élégance (...). C'est le grand réconfort moral de l'heure que de pouvoir rétablir les liaisons avec ses amis, alors que la rupture avec le passé paraît totale». Ayant pris contact en arrivant à Montpellier avec le Professeur Jules Sion, il en avait «reçu l'accueil le plus cordial et le plus généreux [il est, grâce à lui, installé avec sa famille à la Cité Universitaire, alors que beaucoup de réfugiés doivent camper dans les rues]. Il me persuada de me remettre au travail, fit des projets pour une collaboration future et quelques jours après il tombait foudroyé par une attaque subite d'angine de poitrine. Il disparaît ainsi en pleine activité, victime je crois surtout des événements (...). Ce fut pour moi une tristesse de plus, parmi tant d'autres, que d'être le seul géographe à lui rendre un dernier hommage. Je crois que ce fut un homme de grand cour, d'une extrême finesse et un grand géographe, handicapé surtout par une modestie exagéré (...). Mr de Martonne m'a écrit une lettre bien effondrée, très belle, très triste». Les nouvelles des collègues sont rares, celles de Demangeon, resté malade à Paris, très inquiétantes. «La Cité Universitaire possède une vaste terrasse d'où la vue s'étend, splendide, de l'Aigoual à la Méditerranée. La beauté du pays apporte quelques minutes d'oubli. Enfin il me reste une occupation sérieuse: les seuls documents que j'ai pu emporter de Paris sont les tableaux de la carte de croissance de la région parisienne; alors je m'applique à terminer votre ouvrage, petit à petit, sans se presser».

La lettre du $1^{\text {er }}$ août est presque tout entière consacrée au nouveau deuil cruel qui frappe alors Gottmann: la mort de son maître Albert Demangeon, connue avec cinq jours de retard: "Je lui dois en somme tout ce que j'ai appris et tout ce que je suis devenu en géographie. Le début de notre collaboration remonte à novembre 1932 (...). Demangeon est mort, seul, loin de tous ses enfants, en territoire occupé et les gens n'apprendront son décès que plus tard et par hasard. Et ce n'est pas la moindre tristesse de songer qu'il n'aura pas pu terminer les travaux dont il voulait couronner son æuvre. Il m'a parlé à plusieurs reprises (à vous aussi, je crois) de son 'chant du cygne'. Il ne lui aura pas été donné de le chanter (...). Mr de Martonne a bien l'intention de regagner Paris dès qu'il le pourra et de là il me dira si je dois ou non venir. Je serais seul capable d'ailleurs de remettre de l'ordre dans les collections de l'Institut, ayant été le seul à en diriger l'emballage et puis il y a les enquêtes et les dossiers de Demangeon dont je suis maintenant le seul responsable (...). Dans sa dernière lettre Mr de Martonne me demandait votre adresse à Lisbonne: Mme Birot lui avait demandé de vous faire parvenir une lettre; la pauvre est fort inquiète de son mari dont on n'a toujours pas de nouvelles (...). Je serais certes bien heureux de visiter votre 
terrain de thèse et de profiter de votre science de votre pays; mais le moment n'est guère propice aux projets d'avenir (...). Les calculs de la carte sont achevés...Ce gros travail pourra-t-il servir encore à une publication de $\mathrm{Mr}$ Demangeon?».

Orlando Ribeiro a conservé les lettres de E. de Martonne et de Mme Birot, annoncées par Gottmann. La première lettre de cette dernière, du 11 juin, respire l'angoisse. "Vous serait-il possible, au cas où vous connaîtriez quelqu'un dans la diplomatie de votre pays, de savoir si mon mari est dans un camp de prisonniers en Allemagne. Il était lieutenant au ler Bataillon du $148^{e}$ Régiment d'Infanterie de Forteresse, et était en ligne près de Mézières». (Mézières est une ville frontalière, fort exposée en cas de guerre, mais que Birot avait expressément demandée, à cause de sa situation au contact du Bassin sédimentaire parisien et du Massif ancien des Ardennes, un peu comme l'est Coimbra, où il avait commencé à travailler en 1938 et 1939, et où il reviendra, cinq ans plus tard, dès qu'il sera rapatrié d'Allemagne pour raisons de santé, en avril 1944). La lettre de Mme Birot, datée du 25 juillet 1940, est déjà plus sereine: Son mari «a été fait prisonnier le 20 mai après avoir erré plusieurs jours seul dans les bois (...). Transféré dans un camp de prisonniers, en Allemagne, il a retrouvé trois anciens étudiants et organisé, avec eux et d'autres professeurs, une petite université. C'est une manière comme une autre de passer le temps et sa captivité lui semblera moins longue s'il réussit à développer en quelques-uns l'amour de la morphologie».

La lettre suivante de Gottmann est datée du 19 septembre: «Depuis quelques semaines je me suis remis au travail. Il y a à faire dans cette région. Montpellier est une ville des plus séduisantes, les gens y ont un charme 19e siècle, l'Université ancienne et renommée a un charme qui fait songer à l'époque Louis XV et la vieille ville, c'est du XVIIe siècle tout pur. Seulement pour un géographe parisien type 1940 tout cela semble bien endormi, malgré la foule des réfugiés (au fait, il y en a ici d'Espagne, de Hollande, Belgique, Luxembourg, de France et d'autres; comment Bowman peut-il encore croire que les Européens ne savent pas migrer?) (...). De plus j'ai déniché ici à l'École d'Agriculture un professeur de botanique fort intéressant, un vrai géographe manqué, Mr Kuhnholtz-Lordat, qui a fait un livre passionnant sur le rôle du feu en agriculture et ses idées pourraient faire une petite révolution en biogéographie. Avec cela j'ai rencontré ici un vieux savant russe qui connaît fort bien son pays et avec lequel j'ai un peu discuté le problème de l'origine (humaine, climatique ou pédologique?) de la steppe. Ceci me fait penser aussi à la formule de Birot [une formule climatique, liant température et précipitation, que Birot considérait comme la mieux adaptée aux climats méditerranéens]; en auriez-vous quelques nouvelles? Le manque de nouvelles depuis la mi-mai est bien inquiétant.» On note que Gottmann ne savait rien, le 19 septembre, ni de Birot ni de E. de Martonne, alors que la correspondance entre Paris et Lisbonne avait repris presque normalement dès juillet. En effet, une coupure postale pratiquement totale avait été imposée par les Allemands entre 
les zones libre et occupée de la France. Gottmann se demandait avec angoisse ce qui se passait à Paris et quel serait son avenir. "Je commence à être effrayé par la perspective d'une longue période de chômage et des plans variés, hétéroclites, se disputent dans ma tête».

Autre lettre conservée, celle du 24 octobre 1940 (il manque au moins une lettre, dans laquelle Gottmann entretenait déjà Orlando de ses «projets américains»). Gottmann envoie «les détails promis sur la biographie de Demangeon», tels qu'il les a établis avec un fils de celui-ci, professeur au lycée de Montpellier. Cette longue note très détaillée sera utilisée par lui dans l'important article nécrologique qu'il publiera sur son maître, dès 1941, en collaboration avec Pierre Gourou, dans le Bulletin de la Société Languedocienne de Géographie. Pour Orlando, au contraire, le projet d'écrire un texte d'hommage à Demangeon ne se concrétisera jamais, bien qu'il y ait pensé toute sa vie, alors qu'il a consacré le numéro 10 de Finisterra à la mémoire de son autre maître, de Martonne. Le reste de la lettre de Gottmann parle des projets américains: «ils se précisent de plus en plus. J'ai demandé à de Martonne d'essayer de m'obtenir, si c'était encore possible, une mission pour les États-Unis. [De Martonne écrira, en effet, le 10 décembre, une série de lettres à ses collègues américains pour leur recommander Gottmann (Pitte, 2007)]. D'un autre côté on a promis à mon père et à la famille un visa américain pour la durée de la guerre, Mais il est possible que le développement de la nouvelle législation [Un rigoureux «statut des Juifs» venait d'être décrété par le gouvernement de Vichy, le 3 octobre.] et les événements en viennent à nous rendre difficile le séjour en France jus$q u$ 'à l'arrivée de ce visa américain. Alors voilà où j'en viens à vous demander un très grand service: serait-il possible d'obtenir pour notre famille de quatre personnes ou, au moins, pour mes parents seuls, les visas portugais qui nous permettraient éventuellement d'attendre à Lisbonne ce visa américain. (...) Si je me permets d'en appeler ainsi en toute franchise à votre amitié, c'est parce que j'attends de vous la même franchise dans la réponse. Je précise que si je suis de nationalité française, mes parents et mon grand-père sont des apatrides nés en Russie et c'est pour eux encore plus que pour moi que la situation pourrait devenir intenable ici». Mais, en fin de lettre, la Géographie reprend une fois de plus le dessus: Gottmann s'est rendu à Marseille avec un collègue, de Vaumas, et il a visité Arles au retour: "cette ville est un véritable poème; je crois qu'en la quittant je comprenais un peu mieux ce que c'est que Rome, la civilisation latine et l'humanité méditerranéenne. À Montpellier, j'ai commencé à lire un bouquin magnifique: "Un siècle de colonisation», de E. F. Gautier (coll. du Centenaire de l'Algérie). C'est un chef d'œuvre non seulement scientifique mais encore littéraire (...). Oui, il y a eu une belle équipe à l'Institut et je suis bien heureux d'en avoir été. Ce fut une splendeur qui ne reviendra pas de sitôt. Curieux, je parle comme un bien vieux monsieur... et j'ai eu hier 25 ans!».

La lettre suivante est datée du 9 avril 1941, mais plusieurs autres manquent certainement. Gottmann est toujours à Montpellier. Il félicite et console 
Orlando pour sa nomination à Coimbra et non pas à Lisbonne, comme il l'avait espéré: «que vous soyez à Cö̈mbre ou ailleurs, chargé de cours ou titulaire, nous attendons de vous une activité que nous ne jugerons pas à l'étiquette (...). Je tiens à vous remercier pour ce que vous avez fait à la suite de ma demande de décembre et je sais combien toutes ces démarches vaines sont désagréables (...). Toute ma famille (moi y compris) a reçu le visa américain (...). Il reste encore une foule de formalités à remplir». Après avoir énuméré toutes les démarches, avortées pour la plupart, qui ont été faites pour permettre son installation aux États-Unis, Gottmann transmet les nouvelles des géographes français: des morts, des prisonniers, des nominations provisoires. "Le chargé de cours à Montpellier qui enseigne depuis novembre est Gourou, un type de très grande valeur et que j'estime de plus en plus (...). Larnaude est devenu le chef de cabinet de Carcopino, Secrétaire d'Etat à l'Éducation Nationale [du gouvernement dit 'de Vichy', présidé par Pétain et siégeant en zone libre] et $m$ 'a joyeusement annoncé son arrivée à Vichy. Baulig est toujours à Clermont-Ferrand [1'Université de Strasbourg, située dans l'Alsace annexée par les Allemands, avait été repliée à Clermont], Blache [Professeur à Nancy, ville elle aussi annexée par les Allemands] s'est replié à Grenoble, Dion est quelque part en zone occupée». Les revues de Géographie commencent à reparaître, dont un fascicule du Bulletin de la Société Languedocienne de Géographie, que Gottmann a réussi à faire sortir «avec des fragments posthumes de Jules Sion sur la Civilisation Agraire Méditerranéenne, qui me semblent extrêmement intéressants». Après des considérations sur les travaux personnels d'Orlando, il ajoute: «ayant achevé les calculs de la carte 'Région Parisienne' de Demangeon, j'ai dressé la carte elle-même. Le résultat fut intéressant. Un beau-frère du Maître a porté à Paris la carte (que j'ai indiqué avoir été établie par moi en collaboration avec vous). J'espère qu'elle paraîtra un jour dans la G.U. ». Il semble, en fait, que cette carte, qui a tant occupé Gottmann, n'ait jamais été publiée. Elle ne se trouve ni dans La France Économique et Humaine, œuvre posthume de Demangeon, publiée en 1946/48 dans la collection de la Géographie Universelle, ni dans 1'Atlas de France, diffusé à partir de 1947. Gottmann ajoute: "Il n'est pas impossible en fin de compte que je traverse votre pays cet été».

Trois courtes lettres de Gottmann, datées de Lisbonne, sont en effet conservées. Orlando Ribeiro résidait alors à Coimbra, où il enseignait à l'Université. Le 30 octobre 1941 Gottmann le remercie pour «ses propositions» et pour l'avoir mis en contact avec le géologue Zbyszewski, un français d'origine russo-polonaise, installé depuis quelque temps au Portugal. Gottmann doit partir en principe pour l'Amérique le 8 novembre par le paquebot portugais 'Colonial', un vieux bateau des lignes africaines, reconverti en transatlantique. Il essaie de combiner une rencontre et il remercie Orlando, le 3 novembre, de venir le voir à Lisbonne. Il avoue: "Vous n'avez pas idée de l'état dans lequel je suis arrivé ici». N'ayant pas le droit de quitter la France à cause de ses obligations mili- 
taires, il venait en effet de traverser clandestinement le Midi de la France et l'Espagne, avec l'aide de membres d'une 'Résistance' qui commençait alors à s'organiser (Muscarà, 2005: 79-83). Enfin, le 11 novembre, il dit devoir «embarquer dans quelques heures. (...) Les trois jours que vous m'avez consacrés à Lisbonne (...) furent non seulement les plus intéressants et les plus agréables de mon séjour mais encore nos entrevues m'ont sensiblement retapé le moral (...). Maintenant je vais partir pour de vrai, sans savoir quand, comment, ni pourquoi je reviendrai un jour par ici (...). Je pense avec une certaine tristesse au fait que si je publie là-bas quelque chose, il faudra vous l'envoyer... en anglais».

\section{III. À TRAVERS L'ATLANTIQUE}

La lettre suivante, du 5 janvier 1942, est déjà datée de New York, où Gottmann débarqua le 12 décembre, après 22 jours de mer et huit jours de quarantaine à Ellis Island. Les Japonais avaient attaqué la flotte américaine à Pearl Harbour cinq jours plus tôt, précipitant les États-Unis dans la guerre. Gottmann raconte: «je m'américanise. Dès les premiers jours, comme vous le devinez, je me cherche une situation et ce n'est pas commode (...). Si vous avez l'occasion d'écrire à de Martonne, donnez-lui de mes nouvelles, dites que les débuts sont durs mais que je tiendrai le coup et que je pense beaucoup à lui et à l'Institut (...). Si cela ne vous ennuie pas trop pourriez-vous expédier le mot ci-joint à des parents à moi, à Varsovie». Orlando continuait donc, et il continuera pendant des années, à servir de 'boîte postale' entre les pays en guerre.

Le 2 avril 1942, Gottmann informe Orlando qu'il s'est «casé comme vous le voyez par ce papier à en tête. Je suis, depuis ler mars, membre de cet Institute for Advanced Study (cela fait déjà «membre de l'Institut») qui est l'un des meilleurs Instituts de recherche scientifique d'Amérique (...) il n'y a jamais eu de géographe professionnel résidant à Princeton jusqu'ici et cela fait de moi une bête fort curieuse (...) un lieu de travail rêvé (...) selon mon habitude, plus de travail en vue que je n'aurais le temps d'en faire (...). J'avoue n'avoir pas eu encore le temps de vous faire une bibliographie sur la question Pediment [Orlando tentait alors d'interpréter le relief de la Beira Baixa, en postulant l'intervention d'une période d'aridité villafranchienne, qui serait responsable, en autres, des inselberg de Monsanto (Ribeiro, 1942)]. Mais je serai bientôt à l'American Geographical Society (...). Je leur ai montré vos travaux (...).»

Dans sa lettre du 10 avril 1943, la première qui soit écrite à la machine, Gottmann se plaint de n'avoir pas eu de réponse à deux ou trois lettres. Il parle de son «activité effrénée mais où la recherche pure et l'avancement de la science tient vraiment peu de place hélas! Au moins en temps de guerre ai-je la satisfaction morale de ne pas être tout à fait inutile. [Il travaillait alors, tant pour le 
gouvernement et les services d'information militaire américains, que pour la 'France Libre'.] (...). Avez-vous de nouvelles publications? Si vous pouviez me les envoyer, $j$ 'en ferais des comptes rendus dans la Geographical Review (...). Avez-vous eu des nouvelles de nos géographes? (...).»

Une longue lettre d'Orlando est conservée sous la forme d'un brouillon, daté du 5 septembre 1943: "Je vous écris d'un village du Minho où les hasards de l'exploration géographique m'ont conduit pour jouir de quelques jours de repos. Depuis août je n'ai pas reçu de vos nouvelles. Mais vous $m$ 'avez fait parvenir votre bouquin paru au Canada [Gottmann, 1942] (...). Il est, semble$t$-il, d'une très grande opportunité. En même temps, j'y retrouve un peu la manière du Maître (...). Par cette chaude après-midi, après un repas plantureux arrosé de vin vert, je me suis disposé à vous écrire une longue lettre pour vous donner des nouvelles de la géographie portugaise». Orlando conte alors les épisodes qui l'ont amené à transiter de Coimbra à Lisbonne et sans doute est-il encore trop tôt pour transcrire ici le franc-parler avec lequel il juge certains collègues, mais on retiendra déjà diverses indications intéressantes: il espère publier dès l'année suivante une revue et invite Gottmann à y collaborer (en fait, la revue Finisterra ne débutera que 23 ans plus tard, en 1966, grâce aux crédits finalement attribués par la Fondation Gulbenkian, mais le compte-rendu de l'œuvre phare de Gottmann, Megalopolis, y figurera bien dès le premier numéro.) «Si je suis presque seul du côté de la géographie, j'entretiens avec les géologues de Porto et du Service Géologique d'excellentes relations. Je m'y suis mis moi-même, car quand on veut faire de la géomorphologie dans des régions où la géologie n'est pas avancée, il faut faire les deux à la fois». Il évoque aussi ses bonnes relations avec les météorologues et avec le service de la Statistique et annonce un livre en préparation sur La Méditerranée et l'Atlantique dans la Géographie du Portugal. Il pense alors encore achever la thèse de doctorat d'État, commencée avant la guerre. «Les communications avec la France sont presque inexistantes. J'ai vu Deffontaines à Lisbonne et j'ai appris qu'il avait été destitué de son poste à Barcelone. [Pierre Deffontaines, chargé en 1939 de réinstaller un Institut Français à Barcelone, après la guerre civile, refusa de faire allégeance au gouvernement de Vichy et fut chassé de son Institut par la police espagnole; il organisa alors un Institut clandestin, rattaché au gouvernement d'Alger (Delfosse, 1998).] De Martonne continue à travailler d'arrache-pied et son volume sur La France Physique est un véritable monument de clarté, de richesse, de synthèse et de renouveau du sujet. [Ce livre avait été publié à Paris, en 1942, sur du papier soigneusement mis en réserve par l'éditeur, et de Martonne l'avait envoyé à Orlando par des voies détournées, avec une affectueuse dédicace (Ribeiro, 1973: 229)] (...). Quand j'aurai la possibilité de publier dans les Annales [les Annales de Géographie, organe de l'Institut de Géographie de Paris], je ferai sortir un certain nombre d'articles sur la transhumance, la vie montagnarde, la morphologie aride et les céréales au Portugal (...). Je manque presque complètement de bibliographie. Donc, pour l'instant, je fais des mono- 
graphies sur les questions portugaises (...). Nous nous retrouverons à Paris, car je m'y rendrai pour participer à la grande joie de la libération. Pourvu qu'elle ne tarde plus beaucoup.» Mais la 'Libération' tant espérée tardera encore de nombreux mois, les troupes alliées n'étant entrées à Paris que le 24 août 1944.

\section{LE CONGRÈS INTERNATIONAL DE GÉOGRAPHIE DE LISBONNE}

Sans doute Orlando laissa-t-il quelques lettres de Gottmann sans réponse, car il lui écrit, le 2 octobre 1947: «Mon cher Ami, Vous avez toutes les raisons de m'en vouloir et de m'avoir envoyé une lettre très polie et très impersonnelle d'adhésion au Congrès [celle-ci, comme des lettres postérieures de Gottmann, doit se trouver dans les archives du Centro de Estudos Geográficos, malheureusement encore inaccessibles]. Mais quand on a été de si bons amis et quand on s'est quitté dans des circonstances si impressionnantes, on a droit à ne pas maintenir une situation dont je suis d'ailleurs l'unique responsable. Je ne cherche pas à m'excuser, mais plutôt à m'accuser...». Suit un résumé de ce qu'a été la vie personnelle et surtout professionnelle d'Orlando depuis 1943, thème de grand intérêt mais qui trouvera mieux sa place dans une autre publication.

La lettre suivante d'Orlando est du 8 décembre. Écrite entre un voyage à Paris pour préparer le Congrès et un départ en Guinée portugaise, elle montre que les deux amis se sont réconciliés. Orlando consulte longuement Gottmann sur l'organisation du Congrès et désire lui confier diverses responsabilités. «D'accord avec de Martonne, je vous ai fourré secrétaire de la section de Géographie humaine et économique, qui sera présidée par Sorre, Dudley Stamp et un Américain dont j'ai demandé l'indication à Cressey (...) Géographie Coloniale - il paraît que c'est un mot fâcheux et qui froisse les gens (...). Mr Robequain (...) aurait voulu changer le titre en Géographie tropicale, ce qui n'est pas le même (...). Bref, on a changé le titre en Géographie de la Colonisation (...)»». Il discute aussi les problèmes soulevés par la proposition de Gottmann de créer des Commissions traitant de la Géographie de la Circulation et de la Planification Régionale. Relativement à cette dernière, il écrit: «J'adhère avec enthousiasme. Contre l'avis de mes collègues qui tiennent à faire des études géographiques un moyen de se rendre inutiles, $j$ 'ai inclus une question relative aux plans d'aménagement des villes. Il faudrait l'élargir et faire du country planning. Mais c'est plutôt l'affaire d'une commission que l'on pourrait essayer de créer à Lisbonne (...).»

Gottmann lui répond longuement, le 20 décembre 1947. Il discute attentivement les différents thèmes proposés mais demande qu'on le dispense de la plupart des responsabilités: "Je suis, mon cher, vieilli et fatigué; je n'ai plus l'enthousiasme débordant d'antan (...) laissez-moi vous avouer qu'un an et demi passé au Secrétariat International des Nations Unies, m'a laissé une hor- 
reur sacrée de ce genre de travail. Croyez que j'admire votre activité.» Il suggère le nom de divers collègues pour le remplacer, accepte l'appellation de Géographie de la Colonisation, «bien qu'en anglais le processus de colonisation s'appelle 'settlement' quand il s'agit de colonisation de peuplement et 'colonization' lorsqu'il s'agit d'exploitation (...). J'espère qu'un jour il y aura aussi une Géographie de l'indépendance; il y a tant de pays qui s'appellent indépendants aujourd'hui et si peu qui le sont de fait (...). Planification régionale: heureux de voir que nous sommes d'accord (...). Le 'Regional Planning' me paraît devoir être le but et l'aboutissement de la monographie régionale (...). Je suis de plus en plus porté à voir la vanité des régions naturelles en géographie humaine: les zones de civilisation valent bien mieux et sont bien plus intéressantes».

Jean Gottmann sera présent au Congrès (il annonce pour le 6 avril son arrivée à Lisbonne, dans un mot envoyé de Paris le 27 mars 1949, où il met en garde Orlando contre les «étranges projets [des Anglais et des Américains] de constituer un Comité Exécutif de l'Union où il y aura le moins possible de gens parlant autre chose que l'anglais», mais il ne fera pas de communication.

\section{LES CONTACTS LES PLUS RECENTS}

Des vies surchargées de travail, des santés fragiles, font que les rencontres s'espacent et que les lettres se font plus rares. De 1945 à 1968, année où il se fixera en Angleterre, à Oxford, la vie de Jean Gottmann se transforme en ce qu'il appellera avec humour sa 'transhumance atlantique': il ne traversera en effet pas moins de 23 fois l'océan, par bateau ou par avion, pour enseigner alternativement dans de nombreuses universités européennes et américaines, et pour travailler au sein de divers instituts de recherche. Ses efforts pour trouver un emploi stable en France se sont révélés vains, car il n'était, en raison des circonstances, ni 'agrégé' ni 'docteur', alors que ces titres étaient devenus pratiquement indispensables pour espérer entrer dans le cercle étroit, et fort bien défendu, des Professeurs universitaires. Il était, de plus, demeuré paralysé pendant de longs mois, en 1952, après une mauvaise chute (Sur toute cette période, voir en particulier Muscarà, 1998, 2003 et 2005).

Le 10 novembre 1949, Gottmann dédicaçait à Orlando, «en toute amitié», son livre sur L'Amérique. Le 28 juin 1953, il lui envoyait du paquebot l'Ile de France, en route vers New York, une carte postale lui rappelant, depuis les «brumes de Terre Neuve (...) votre bonne promesse de quelques données pour mon Europe» [l'édition révisée de A Geography of Europe, qui sera publiée à New York, en 1954]. En janvier 1962, Gottmann, lors d'un de ses séjours en Europe, essaya de combiner avec Orlando, alors Professeur visitant au Québec, une rencontre à Paris ou à Lisbonne. Cette tentative échouera, par ma faute je le crois bien, puisque, au retour du Canada, Orlando viendra 
découvrir avec moi le Finistère breton qu'il ne connaissait pas encore et dont il utilisera les photos dans son livre Geografia e Civilização, prédaté de 1961. Il rentrera ensuite précipitamment de France au Portugal, pour ne pas être absent des célèbres 'balburdias' universitaires, qui venaient d'éclater à Lisbonne (Ribeiro, 1976: 126).

Mais, si la partie aujourd'hui connue de la correspondance entre les deux amis s'achève avec une carte postale envoyée le 13 juillet 1973 de Macau par Gottmann, les relations intellectuelles ne cessèrent pas. Dès le premier numéro de Finisterra, on l'a déjà dit, une place avait été réservée à un soigneux compte rendu de Megalopolis (1961) par Ilídio do Amaral (1966). Celui-ci y montrait non seulement l'énorme richesse conceptuelle et factuelle de cette œuvre majeure de Gottmann, mais il indiquait aussi quel rapide impact elle avait déjà eu aux États-Unis et en France.

La contribution que Gottmann envoya, dès le 16 août 1980, au même Professeur Ilídio do Amaral pour le Livro de Homenagem a Orlando Ribeiro et qui sera publiée dans son premier volume (1984), est un dense et lumineux message adressé aux géographes portugais. Il devrait, aujourd'hui encore, leur servir d'excellente introduction à la pensée éclairante et solide de Gottmann. Sous le titre Heureux qui comme Ulysse... Réseaux et régions en Géographie humaine, il y montre comment, pour comprendre vraiment les «cas particuliers» qui sont objets d'étude, ceux-ci doivent être impérativement replacés dans les «réseaux de relations extérieures, à tous les niveaux (du climatique au politique et financier)».

Cette lecture introductive à une œuvre très vaste (lui-même l'évaluait, dès 1970, à plus de 5000 pages) peut désormais être utilement complétée par l'excellente auto synthèse, Exposé sur l'ensemble de mes æuvres publiées, que Jean Gottmann avait remise aux quinze collègues français du jury qui lui conféra en 1970, à Nanterre, un bien tardif doctorat d'hommage. Ce texte fondamental vient en effet d'être, pour la première fois, publié (L'Orbite ..., 2007: 274297) et on trouvera, en outre, la liste complète de œuvres de Gottmann, établie par Luca Muscarà, dans Ekistics (2003). Je ne saurais trop recommander à tous les jeunes géographes, quelle que soit leur spécialisation, qui souhaiteraient aborder l'œuvre si éclairante et si actuelle de Gottmann, de lire d'abord les quelques pages écrites en 1970, qui résument lucidement la trajectoire intellectuelle d'un des plus grands géographes du XXe siècle, en évoquant ses efforts pour «arriver à une compréhension meilleure (...) de la géographie du monde», sans oublier une ultime notation: «J'ai le sentiment que je n'ai pas encore atteint l'issue du tunnel dans lequel je me suis ainsi engagé. Il reste beaucoup à éclaircir». 


\section{BIBLIOGRAPHIE}

Amaral I (1966) Megalopolis. Finisterra - Revista Portuguesa de Geografia, 1(I): 131-135.

Daveau S, Feijão M J, Barata P (2002) O espólio científico do Professor Orlando Ribeiro. Finisterra - Revista Portuguesa de Geografia, 73 (XXXVII): 133-137.

Delfosse C (1998) Le rôle des institutions culturelles et des missions à l'étranger dans la circulation des idées géographiques. L'exemple de la carrière de Pierre Deffontaines. Finisterra Revista Portuguesa de Geografia, 65 (XXXIII): 147-158.

Demangeon A (1946/48) La France Économique et Humaine. Armand Colin, Paris.

Gottmann J (2007) Exposé sur l'ensemble de mes œuvres publiées, en introduction à ma soutenance du doctorat d'État (1970, inédit). In L'Orbite de la Géographie de Jean Gottmann, Actes du Colloque International, Paris, mars 2005, La Géographie, n. ${ }^{\circ}$ spécial 1523 bis, Paris, 2007: 274-277.

Gottmann J (1984) Heureux qui comme Ulysse... Réseaux et régions en Géographie humaine. In Livro de Homenagem a Orlando Ribeiro, Vol. I, Centro de Estudos Geográficos, Lisboa: 99$-106$.

Gottmann J (1961) Megalopolis. The Urbanized Northeastern Seaboard of the United States. Twentieth Century Fund, New York.

Gottmann J (1950) A Geography of Europe. Henry Holt, New York; (1954) revised edition.

Gottmann J (1949) L'Amérique. Hachette, Paris.

Gottmann J (1942) Les relations commerciales de la France. France for Ever, 1, Les Éditions de l'Arbre, Montréal.

Gottmann J, Gourou P (1941) Demangeon A (1872/1940). Bulletin de la Société Languedocienne de Géographie, Montpellier, 12: 1-15.

Martonne E (1942) La France Physique. Armand Colin, Paris.

Martonne E (1925/27) Traité de Géographie Physique. Armand Colin, Paris (3 volumes, 4e édition).

Muscarà C (2003, ed.) In the Steps of Jean Gottmann. Ekistics, 70, 418/19, 420/21, 422/24.

Muscarà L (2005) La Strada di Jean Gottmann. Tra universalismi della storia e particolarismi della geografia. Nexta Books, Roma.

Muscarà L (2003) The long road to Megalopolis. Ekistics, 70, 418/19: 23-35; The complete bibliography of Jean Gottmann, Id.: 111-119.

Muscarà L (1998) Jean Gottmann's Atlantic 'Transhumance' and the development of his spatial theory. Finisterra - Revista Portuguesa de Geografia, XXXIII (65): 159-172.

Pitte J-R (2007) Allocution d'accueil à la Sorbonne. In L'Orbite de la Géographie de Jean Gottmann, Actes du Colloque International, Paris, mars 2005, La Géographie, n. ${ }^{\circ}$ spécial 1523 bis, Paris, 2007: 6-8.

Ribeiro O (1976) A Universidade em Crise. Edições Cosmos, Lisboa.

Ribeiro O (1973) Um mestre da Geografia do nosso século - Emmanuel de Martonne (1873/1955). Finisterra - Revista Portuguesa de Geografia, V (10): 163-164.

Ribeiro O (1961) Geografia e Civilização. Temas portugueses. Chorographia, Centro de Estudos Geográficos, Lisboa (3édition, 1992).

Ribeiro O (1942) Notas sobre a evolução morfológica da Orla meridional da Cordilheira Central, entre Sobreira Formosa e a fronteira. Boletim da Sociedade Geológica de Portugal, Porto, I (3): 5-27. 
Sarazin J-Y (2007) Le don de Jean Gottmann à la Bibliothèque nationale de France: des sources pour éclairer ses travaux et sa personnalité. In L'Orbite de la Géographie de Jean Gottmann, Actes du Colloque International, Paris, mars 2005, La Géographie, n. ${ }^{\circ}$ spécial 1523 bis, Paris, 2007: 17-28.

L'Orbite de la Géographie de Jean Gottmann, Actes du Colloque International, Paris, mars 2005, La Géographie, n. ${ }^{\circ}$ spécial 1523 bis, Paris, 2007.

Voyage, Circulation et Transfert d'Idées Géographiques, Symposium, Lisboa, 27-29 août 1998, Finisterra - Revista Portuguesa de Geografia, XXXIII (65), 1998: 11-228. 